\title{
Nonequilibrium Alfvénic Plasma Jets Associated with Spheromak Formation
}

\author{
Deepak Kumar and Paul M. Bellan \\ California Institute of Technology, Pasadena, California 91125, USA
}

(Received 6 February 2009; published 4 September 2009)

\begin{abstract}
Nonequilibrium Alfvénic flows have been observed in plasma jets during the helicity injection stage of the Caltech spheromak experiment. Density and time of flight measurements of these jets show that the flows convect dense plasma $(\beta \sim 1)$ because of the axial gradient in the current channel profile. A simplified MHD theory is derived to model the flow.
\end{abstract}

DOI: 10.1103/PhysRevLett.103.105003

MHD-driven flows have been observed over a wide range of scales from terrestrial experiments (coaxial gun accelerators [1,2], plasma thrusters [3], high-current arcs [4], Z-pinch formation [5], spheromak formation [6,7], and sustainment [8]) to extraterrestrial phenomenon (solar coronal mass ejections [9] and astrophysical jets [10]).

Various mechanisms and scalings for these flows have been proposed. For example, Reed [4] proposed a mechanism whereby the axial flow velocity $u$ should scale as $I^{1 / 2}$ in flared high-current arcs, where $I$ is the arc current. As another example, Barnes et al. [8] used a zero-pressure plasma model to predict that the flow of plasma from the electrodes in a steady-state driven spheromak should scale as $I^{3}$. This Letter presents quantitative measurements of MHD-driven flow velocity and shows that $u \sim I$. A model is presented showing that flow results from a process whereby the radial magnetic pinch force associated with I produces a large on-axis plasma pressure. Axial nonuniformity of the current channel results in a plasma pressure that is largest near the electrodes. The axial gradient of the pressure drives plasma jet flow away from the electrodes.

This Letter also has important implications regarding the classic Taylor relaxation theory [11] conventionally used to model spheromaks, reversed field pinches, and aspects of solar coronal loops [12]. Our experimental results show that evolution of the magnetized plasma is not via a sequence of zero-pressure gradient static equilibria [6] as presumed in the Taylor model, but rather involves large pressure gradients and fast flows.

The Caltech spheromak experiment $[13,14]$ has a cylindrical geometry with coaxial planar electrodes for helicity injection (see Fig. 1). A vacuum magnetic field created by a coil behind the electrodes links an inner disk cathode and outer annular anode. Just before the discharge, neutral gas is puffed near the electrodes using 16 orifices, eight each on the two electrodes. A capacitor bank $(59 \mu \mathrm{F}, 6-8 \mathrm{kV})$ is switched across the electrode by an ignitron to create the discharge. The experiment normally uses two $59 \mu \mathrm{F}$ capacitors in parallel, but only one capacitor was used for the measurements reported in this Letter. This was done to eliminate jitter associated with firing two ignitrons and also to limit the current below the plasma kinking threshold [13]. As seen from typical current and voltage traces
PACS numbers: 52.30.Cv, 52.55.Ip, 52.55.Wq, 52.72.+v

(Fig. 2), the current and voltage across the electrodes are approximately out of phase. This implies that the plasma can be considered an inductive load. Figure 1 shows a series of plasma images which elucidate the sequence of plasma evolution leading to a changing inductance. Initially ( $\sim 0.5 \mu$ s after breakdown) eight "spider legs" are formed linking the gas nozzles on the two electrodes. Previous work [14] has primarily focused on the collimation and flow of plasma in these spider legs. As the current ramps up, the spider legs expand due to hoop force and then merge to form a central column jet because of the pinch force $(\sim 3 \mu$ s after breakdown). This results in a slightly flaring plasma jet which drives plasma from near the electrodes to the vacuum. As the jet evolves outward, it increases the plasma inductance and thus acts as a helicity injection mechanism. This Letter shows that MHD-driven flows act as the mechanism to drive the change in the inductance of the plasma jet. As the jet expands towards vacuum, it eventually overcomes the Kruskal-Shafranov kink

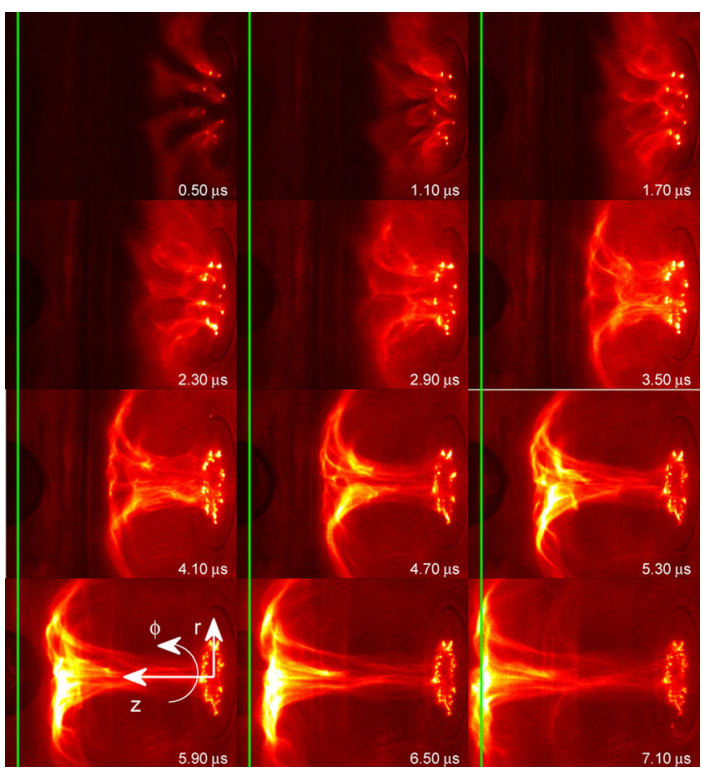

FIG. 1 (color online). False colored images depicting the formation of a hydrogen plasma jet from shot numbers 9920 and 9923. The green vertical line represents the path of the laser beam used to measure plasma density. 


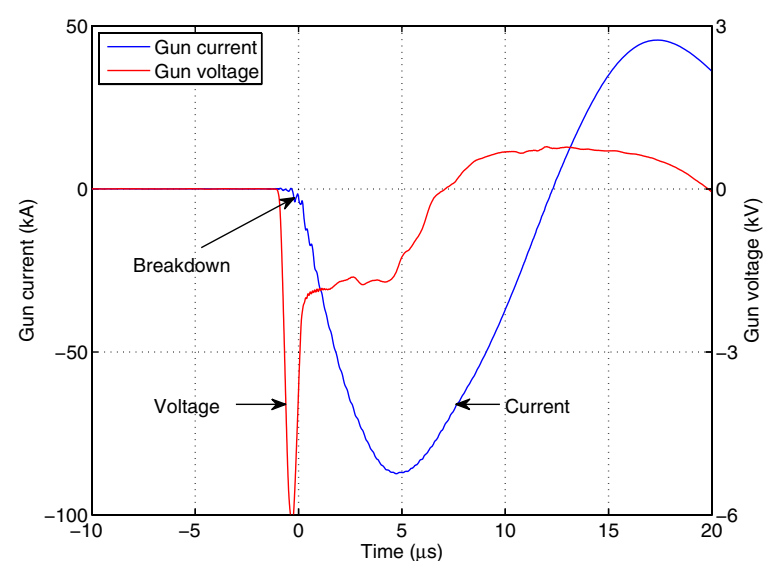

FIG. 2 (color online). Current and voltage trace of plasma jet from shot number 9114.

instability condition (due to an increased axial length), and can detach to form a spheromak-like configuration [13].

A cylindrical coordinate system $\{r, \phi, z\}$ is used in this Letter. The origin is at the center of the electrodes, and the $z$ axis is along the direction of the jet flow (see Fig. 1 at $5.9 \mu \mathrm{s}$ ). The term "poloidal" will be used to refer to the $r$, $z$ component of a vector, and "toroidal" to the component along $\hat{\phi}=r \nabla \phi$.

A He-Ne interferometer [15] was used to measure the density of the plasma jet. The interferometer beam (shown as a vertical green line in Fig. 1) intercepted the plasma jet at a distance of $z=29 \mathrm{~cm}$ from the planar electrodes.

Figure 3 shows typical line-averaged density traces from this interferometer for hydrogen and deuterium plasma jets. From visible images, the radius of the jet is estimated to be about $3 \mathrm{~cm}$ (see Fig. 1), and so the nominal density of the jets is $\sim 3 \times 10^{22} / \mathrm{m}^{3}$.

An obvious characteristic of the density traces shown in Fig. 3 is the extremely sharp rise time in the observed density as the apex of the plasma jet traverses the path intercepted by the laser beam. This sharp rise time gives a time of flight measurement and can be used to estimate the average velocity of the plasma as $v=\frac{L=29 \mathrm{~cm}}{\text { time of flight }}$.

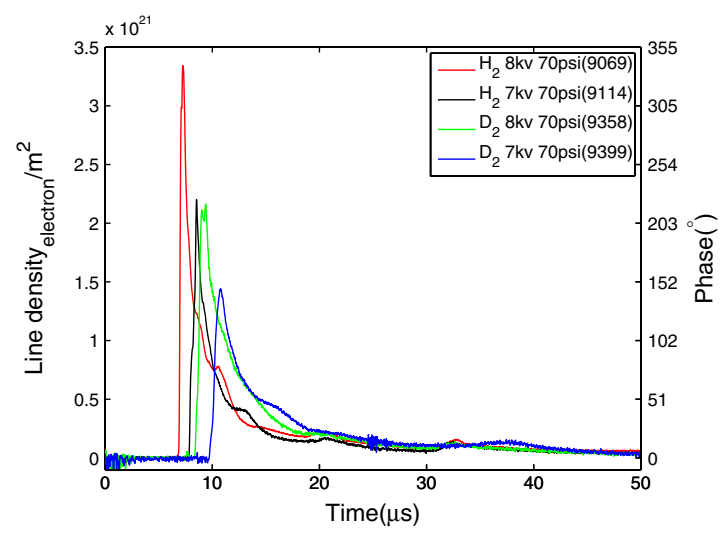

FIG. 3 (color online). Typical interferometer density traces from the plasma jets.

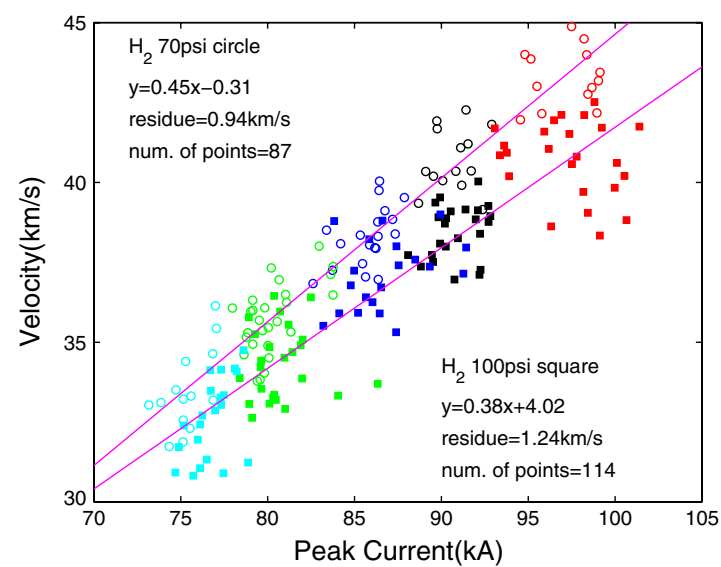

FIG. 4 (color online). Velocity of plasma jet as a function of maximum gun current for $H_{2}$ plasmas. Measurements from shots when the fast gas valves were pressurized to 70 and 100 psi are plotted as circles and squares, respectively. Here, and in subsequent figures, the cyan, green, blue, black, and red data points refer to the gun discharge voltage of $6,6.5,7,7.5$, and $8 \mathrm{kV}$, respectively. Also, the linear fit to the respective data points is plotted in magenta, and the equation for the linear fit and corresponding error is shown next to the lines.

Figure 4 plots the time of flight velocity of hydrogen plasma jets as a function of the maximum gun current flowing through the jet. Plasma experiments were done with fast gas puff valves pressurized with $\mathrm{H}_{2}$ at either 70 or 100 psi. Because Fig. 4 shows that plasma jets with 70 psi gas valve pressure are faster than jets with 100 psi gas valve pressure, the neutral gas pressure gradient cannot be the force that drives the plasma jet.

Figure 5 plots the average velocity of hydrogen and deuterium plasma jets as a function of maximum gun current flowing through the plasma. This shows that the velocity of the plasma jet is proportional to the current flowing through the plasma and that the hydrogen plasma jets are faster than the deuterium ones.

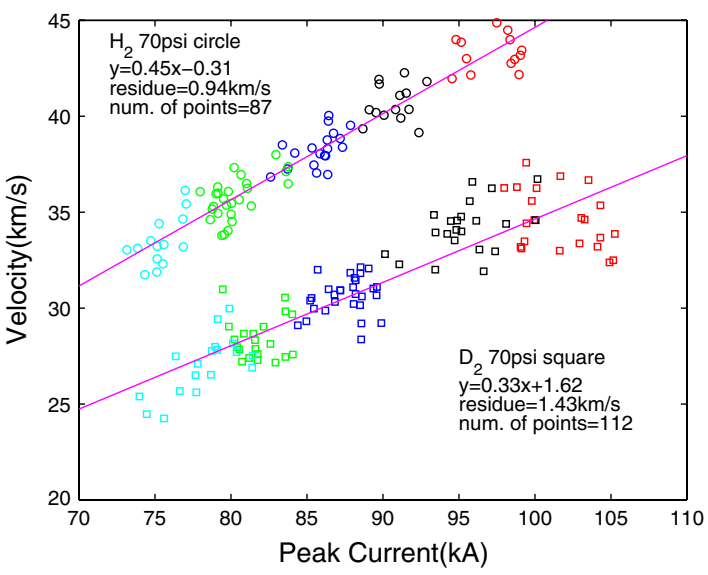

FIG. 5 (color online). Velocity of plasma jet as a function of maximum gun current for $\mathrm{H}_{2}$ and $\mathrm{D}_{2}$ plasmas plotted in circles and squares, respectively. The fast gas valves were pressurized to 70 psi. 
Figure 6 shows that plasma pressure is proportional to the toroidal magnetic field energy density and that the plasma jets have $\beta_{\phi}=\frac{n k\left(T_{i}+T_{e}\right)}{B_{\phi}^{2} / 2 \mu_{0}} \sim 1$. For thermal energy density $n k\left(T_{i}+T_{e}\right)$ in Fig. 6 , ion and electron temperatures were assumed to be $2 \mathrm{eV} \mathrm{[16]} \mathrm{for} \mathrm{all} \mathrm{the} \mathrm{shots,} \mathrm{and}$ density was inferred from the peak of density traces (see Fig. 3) assuming a plasma radius of $10 \mathrm{~cm}$. Toroidal field energy density $\left[B_{\phi}^{2} /\left(2 \mu_{0}\right)\right]$ was calculated using $B_{\phi}=$ $\mu_{0} I /(2 \pi a)$, where $I$ is the instantaneous current flowing through the plasma when the density was measured, and $a$, the radius of current channel was assumed to be $10 \mathrm{~cm}$. The choice of radius was motivated by magnetic probe [17] measurements showing that the current flows through a radius of about $10 \mathrm{~cm}$. Also the typical axial field in the experiment was $B_{z} \sim 0.2 \mathrm{~T}$, which corresponds to $\beta_{z}=$ $\frac{n k\left(T_{i}+T_{e}\right)}{B_{z}^{2} / 2 \mu_{0}} \sim 0.2-0.4$. Plasma density was observed to scale directly with $B_{\phi}$ and inversely with $B_{z}$.

We now present a model showing that the flow is driven by the axial gradient in $B_{\phi}^{2}$ associated with the slight flaring of the jet. This model is a generalization of the model presented by Bellan [18]. In the model, the jet is assumed to be axisymmetric and slightly flared. Furthermore, the poloidal flux inside the jet is assumed to have the simplest nontrivial physically relevant form

$$
\psi(r, z)=\psi_{0} r^{2} / a(z)^{2},
$$

where

$$
a(z)=a_{0} e^{\kappa z}
$$

describes the flaring of the jet of radius $a(z)$. Here $a_{0}$ is the jet radius at the electrodes (refer to Fig. 1), and $\kappa$ is determined from flaring in Fig. 1. The poloidal flux in narrow astrophysical jets has previously been modeled by a similar expression $[19,20]$.

We assume that the pressure $P(r, z)$ vanishes at $r=a(z)$ and that $B_{z}$ is nearly uniform for $r<a$ or equivalently that the radial scale length of $B_{z}$ exceeds the radial scale length for pressure. Plasma jets with large aspect ratio

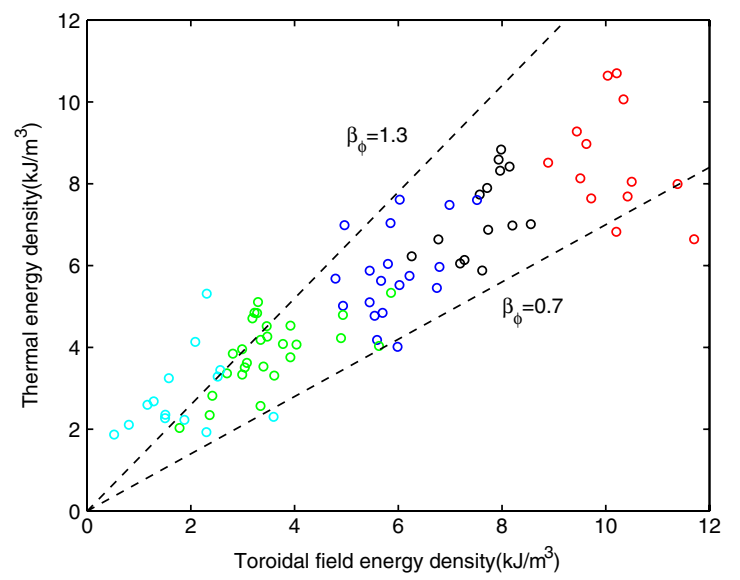

FIG. 6 (color online). Thermal energy density as a function of toroidal magnetic field energy density for $\mathrm{H}_{2}$ plasma jets. (length $\gg$ radius) are assumed to be well described by Eq. (1).

Let

$$
I(r, z)=I(\psi)=\frac{\lambda \psi}{\mu_{0}}=\frac{\lambda \psi_{0}}{\mu_{0}} \frac{r^{2}}{a(z)^{2}}=I_{0} \frac{r^{2}}{a(z)^{2}} .
$$

The assumption $I=I(\psi)$ implies that current flows along flux surfaces so there is no torque $[\hat{\phi} \cdot(\mathbf{J} \times \mathbf{B})]$ causing acceleration in the $\phi$ direction [18].

The associated toroidal or poloidal magnetic fields and current densities are

$$
\begin{gathered}
\mathbf{B}_{\text {tor }}=\frac{\mu_{0} I}{2 \pi} \nabla \phi, \\
\mathbf{B}_{\text {pol }}=\frac{1}{2 \pi} \nabla \psi \times \nabla \phi, \\
\mathbf{J}_{\text {tor }}=-\frac{r^{2}}{2 \pi \mu_{0}} \nabla \cdot\left(\frac{1}{r^{2}} \nabla \psi\right) \nabla \phi, \\
\mathbf{J}_{\mathrm{pol}}=\frac{1}{2 \pi} \nabla I \times \nabla \phi .
\end{gathered}
$$

Because of the jet's large aspect ratio, radial equilibrium is achieved much faster than axial equilibrium. This is evident from Fig. 1, where the radial profile of the jet hardly changes as it evolves. MHD radial pressure balance then implies

$$
\begin{aligned}
\frac{\partial P}{\partial r}= & \left(\mathbf{J}_{\mathrm{pol}} \times \mathbf{B}_{\mathrm{tor}}\right)_{r}+\left(\mathbf{J}_{\mathrm{tor}} \times \mathbf{B}_{\mathrm{pol}}\right)_{r} \\
= & -\frac{\mu_{0}}{(2 \pi r)^{2}} \frac{\partial}{\partial r}\left(\frac{I^{2}}{2}\right)-\frac{1}{(2 \pi r)^{2} \mu_{0}}\left[r \frac{\partial}{\partial r}\left(\frac{1}{r} \frac{\partial \psi}{\partial r}\right)\right. \\
& \left.+\frac{\partial^{2} \psi}{\partial z^{2}}\right] \frac{\partial \psi}{\partial r} .
\end{aligned}
$$

Note that $\frac{\partial}{\partial r}\left(\frac{1}{r} \frac{\partial \psi}{\partial r}\right)=0$ because of the $\psi \sim r^{2}$ dependence assumed in Eq. (1). This implies

$$
\begin{aligned}
\frac{\partial P}{\partial r} & =-\frac{\lambda^{2}}{(2 \pi r)^{2} \mu_{0}} \psi \frac{\partial \psi}{\partial r}-\frac{1}{(2 \pi r)^{2} \mu_{0}} \frac{\partial^{2} \psi}{\partial z^{2}} \frac{\partial \psi}{\partial r} \\
& =-\frac{\left(\lambda^{2}+4 \kappa^{2}\right)}{(2 \pi r)^{2} \mu_{0}} \psi \frac{\partial \psi}{\partial r} .
\end{aligned}
$$

Equation (9) can be integrated radially to give

$$
P(r, z)=\frac{\left(\lambda^{2}+4 \kappa^{2}\right) \psi_{0}^{2}}{(2 \pi a)^{2} \mu_{0}}\left(1-\frac{r^{2}}{a^{2}}\right),
$$

where the boundary condition is $P(r, z)=0$ at $r=a(z)$.

Current and hence $\lambda$ are time dependent in the experiment (see Fig. 2). However, using an average value of current $I \sim 75 \mathrm{kA}$ and applied poloidal flux, $\psi_{0} \sim$ $4 \mathrm{mWb}$ yields a nominal value of $\lambda \sim 20 \mathrm{~m}^{-1}$. From the visual images (see Fig. 1) the flaring of the jet corresponds to $\kappa \sim 2 \mathrm{~m}^{-1}$. Thus $\lambda^{2} \gg 4 \kappa^{2}$ and hence $4 \kappa^{2}$ can be neglected in Eq. (10). Thus, $P(r, z)$ is predominantly determined by the axial current (or equivalently the toroidal magnetic field). For such a jet, the plasma toroidal beta $\beta_{\phi}$ should be of order unity, as shown in Fig. 6. 
Assuming that the plasma has uniform temperature, Eq. (10) implies that $\rho(r=0, z) \sim P(r=0, z) \sim$ $1 / a(z)^{2}$. Thus, the axial dependence of the plasma mass density at $r=0$ can be expressed as

$$
\rho(r=0, z)=\rho_{0} e^{-2 \kappa z} .
$$

The jet is in radial force balance, but there is no such balance along the $z$ axis. Consider the $z$ component of the MHD equation of motion in steady-state

$$
\rho(\mathbf{u} \cdot \nabla) u_{z}=\left(\mathbf{J}_{\mathrm{pol}} \times \mathbf{B}_{\text {tor }}\right)_{z}+\left(\mathbf{J}_{\mathrm{tor}} \times \mathbf{B}_{\mathrm{pol}}\right)_{z}-\frac{\partial P}{\partial z} .
$$

Using Eqs. (1)-(7) and (10), Eq. (12) becomes

$$
\rho(\mathbf{u} \cdot \nabla) u_{z}=-\frac{\partial}{\partial z}\left[\frac{\left(\lambda^{2}+4 \kappa^{2}\right) \psi_{0}^{2}}{(2 \pi a)^{2} \mu_{0}}\left(1-\frac{r^{2}}{2 a^{2}}\right)\right] .
$$

Using $\lambda^{2} \gg 4 \kappa^{2}$, Eq. (13) can be rewritten as

$$
\begin{aligned}
\rho(\mathbf{u} \cdot \nabla) u_{z} & =-\frac{\partial}{\partial z}\left[\frac{\lambda^{2} \psi_{0}^{2}}{(2 \pi a)^{2} \mu_{0}}\left(1-\frac{r^{2}}{2 a^{2}}\right)\right] \\
& =-\frac{\partial}{\partial z}\left[\frac{\mu_{0} I_{0}^{2}}{4 \pi^{2} a^{2}}\left(1-\frac{r^{2}}{2 a^{2}}\right)\right] .
\end{aligned}
$$

Evaluating at $r=0$ gives

$$
\begin{aligned}
\rho_{0} e^{-2 \kappa z} \frac{\partial}{\partial z}\left(\frac{u_{z}^{2}}{2}\right) & =-\frac{\partial}{\partial z}\left(\frac{\mu_{0} I_{0}^{2} e^{-2 \kappa z}}{\left(2 \pi a_{0}\right)^{2}}\right), \\
\frac{\partial}{\partial z}\left(\frac{u_{z}^{2}}{2}\right) & =2 \kappa \frac{\bar{B}_{\phi}^{2}}{\mu_{0} \rho_{0}},
\end{aligned}
$$

where $\bar{B}_{\phi}=B_{\phi}\left(r=a_{0}, z=0\right)=\mu_{0} I_{0} / 2 \pi a_{0}$ is the toroidal magnetic field at the edge of the jet near the electrodes. The equation above can be integrated from $z=0$ to $z=L$ to give

$$
\begin{aligned}
& u_{z}(r=0, z=L) \sim \sqrt{\frac{\bar{B}_{\phi}^{2}}{\rho_{0} \mu_{0}} 4 \kappa L,} \\
& u_{z}(r=0, z=L) \sim \frac{1}{\pi a_{0}} \sqrt{\frac{\mu_{0} \kappa L}{\rho_{0}}} I_{0},
\end{aligned}
$$

where the axial velocity near the electrodes was assumed to be small. Equation (16) shows that the collimated plasma jets are accelerated by magnetic forces, as has been suggested for astrophysical jets $[19,20]$. Note that these laboratory jets do not have any appreciable toroidal velocity.

We now compare the experimental results to the quantitative predictions of the theory. Using typical parameters of a hydrogen plasma jet $\left(n_{e} \sim 3 \times 10^{22} / \mathrm{m}^{3}, a_{0} \sim 3-10 \mathrm{~cm}\right.$, $\kappa \sim 2 \mathrm{~m}^{-1}, L=0.29 \mathrm{~m}$ ), Eq. (16) predicts that the slope of the $u$ vs $I$ linear fit for hydrogen plasmas in Fig. 5 should be $0.38-1.28 \mathrm{~m} \mathrm{~s}^{-1} \mathrm{~A}^{-1}$. The experimentally observed linear dependence has a slope of $0.45 \mathrm{~m} \mathrm{~s}^{-1} \mathrm{~A}^{-1}$. Equation (16) also suggests that the slope for the linear fits in Fig. 5 should scale inversely with the square root of the mass of ions. Thus it predicts that the ratio of slopes of the linear fits in Fig. 5 for hydrogen and deuterium plasmas should be $\sqrt{\frac{1}{2}}=0.707$. From the experiments the ratio of slopes is measured as $\frac{0.33}{0.45}=0.733$. Thus the experimentally measured value agrees reasonably well with the predicted ion mass dependence. The results clearly show that the phenomenon causing bulk plasma motion is nonequilibrium dynamics as the response of the plasma is inversely related to the square root of the ion mass.

It is important to note that the scaling described by Eq. (16) is Alfvénic in a formal sense only and that, in general, the jet is not traveling at the local Alfvén velocity. This is because the jet velocity on-axis scales as $B_{\phi}(r=a, z) / \sqrt{\rho(r=0, z)}$ whereas the local on-axis Alfvén velocity scales as $B_{z}(r=0, z) / \sqrt{\rho(r=0, z)}$.

This Letter demonstrates the existence of Alfvénic jets in situations where there is both poloidal current $I$ and poloidal flux $\psi$; these finite magnetic helicity situations pertain to spheromaks, astrophysical jets, and solar coronal phenomena.

The authors thank D. Felt, A. Moser, and R. Perkins for technical help. Supported by U.S.-DOE and NSF.

[1] J. Marshall, Phys. Fluids 3, 134 (1960).

[2] T. D. Butler, I. Henins, F. C. Jahoda, J. Marshall, and R. L. Morse, Phys. Fluids 12, 1904 (1969).

[3] K. Schoenberg, R. Gerwin, I. Henins, R. Mayo, J. Scheuer, and G. Wurden, IEEE Trans. Plasma Sci. 21, 625 (1993).

[4] T. B. Reed, J. Appl. Phys. 31, 2048 (1960).

[5] U. Shumlak, B. A. Nelson, and B. Balick, Astrophys. Space Sci. 307, 41 (2007).

[6] W.C. Turner, G. C. Goldenbaum, E. H. A. Granneman, J. H. Hammer, C. W. Hartman, D. S. Prono, and J. Taska, Phys. Fluids 26, 1965 (1983).

[7] T. Uyama, Y. Honda, M. Nagata, M. Nishikawa, A. Ozaki, N. Satomi, and K. Watanabe, Nucl. Fusion 27, 799 (1987).

[8] C. W. Barnes, T. R. Jarboe, G. J. Marklin, S. O. Knox, and I. Henins, Phys. Fluids B 2, 1871 (1990).

[9] J. Chen and J. Krall, J. Geophys. Res. 108, 1410 (2003).

[10] M. Nakamura, H. Li, and S. Li, Astrophys. J. 656, 721 (2007).

[11] J. B. Taylor, Phys. Rev. Lett. 33, 1139 (1974).

[12] D. M. Rust and A. Kumar, Astrophys. J. Lett. 464, L199 (1996).

[13] S. C. Hsu and P. M. Bellan, Phys. Rev. Lett. 90, 215002 (2003).

[14] S. You, G. S. Yun, and P. M. Bellan, Phys. Rev. Lett. 95, 045002 (2005).

[15] D. Kumar and P. M. Bellan, Rev. Sci. Instrum. 77, 083503 (2006).

[16] G. S. Yun, Ph.D. thesis, California Institute of Technology, 2007.

[17] C. A. Romero-Talamás, P. M. Bellan, and S. C. Hsu, Rev. Sci. Instrum. 75, 2664 (2004).

[18] P. M. Bellan, Phys. Plasmas 10, 1999 (2003).

[19] R. V.E. Lovelace, H.L. Berk, and J. Contopoulos, Astrophys. J. 379, 696 (1991).

[20] T. Koupelis and H. M. van Horn, Astrophys. J. 342, 146 (1989). 